\title{
Detector dependency of diffusive quantum monitorings
}

\author{
Shakib Daryanoosh, ${ }^{1, *}$ Howard M. Wiseman, ${ }^{1, \dagger}$ and Jay M. Gambetta ${ }^{2, \ddagger}$ \\ ${ }^{1}$ Centre for Quantum Computation and Communication Technology (Australian Research Council), \\ Centre for Quantum Dynamics, Griffith University, Brisbane, Queensland 4111, Australia \\ ${ }^{2}$ IBM T. J. Watson Research Center, Yorktwon Heights, New York 10598, USA
}

(Dated: May 4, 2015)

\begin{abstract}
Continuous measurements play a pivotal role in the study of dynamical open quantum systems. 'Dyne' detections are among the most widespread and efficient measurement schemes, and give rise to quantum diffusion of the conditioned state. In this work we study under what conditions the detector dependency of the conditional state of a quantum system subject to diffusive monitoring can be demonstrated experimentally, in the sense of ruling our any detector-independent pure-state dynamical model for the system. We consider an arbitrary number $L$ of environments to which the system is coupled, and an arbitrary number $K$ of different types of dyne detections. We prove that non-trivial necessary conditions for such a demonstration can be determined efficiently by semidefinite programming. To determine sufficient conditions, different physical environmental couplings and Hamiltonians for a qubit, and different sets of diffusive monitorings are scrutinized. We compare the threshold efficiencies that are sufficient in the various cases, as well as cases previously considered in the literature, to suggest the most feasible experimental options.
\end{abstract}

PACS numbers: 03.65.Yz, 03.65.Ta, 03.65.Aa, 42.50.Dv, 42.50.Lc

\section{INTRODUCTION}

One of the most challenging tasks in the analysis of an open quantum system is to gain as much information as possible about the system state. The conventional approach of tracing over the environment, with which the system is coupled to, would not yield to the maximal knowledge. However, continuous measurements (also referred to as monitorings) of the environment make it possible to gain further information about the system by exploiting quantum correlations between the two [1]. The former approach results in a master equation (ME) for the state matrix $\rho$ of the system whereas the latter gives a stochastic master equation (SME) describing evolution of a conditional state matrix $\varrho$ given a readout. The latter is known as an unraveling of the ME [2], because the ME is recovered by averaging over all possible measurement records. Under ideal conditions, for every single measurement record there is a path of a ray in the system's Hilbert space, called a quantum trajectory.

A SME may have more than one physical realization (that is, the set of different ways of monitoring the environment is a surjective mapping onto the set of all possible SMEs). In the context of quantum optics, different monitorings of the environment can be achieved by adding a local oscillator (LO) to the system output field prior to detection. Depending on the strength, frequency, and phase of the LO in comparison with those of the emitted radiation the dynamics of the system are described by distinct SMEs. These fall into two categories: jump unravelings and diffusive unravelings [1]. For quantum

\footnotetext{
* s.daryanoosh@griffith.edu.au

† h.wiseman@griffith.edu.au

‡ jay.gambetta@us.ibm.com
}

jumps the LO either is a weak field or is set at zero (direct detection), while for quantum diffusion it is a strong field, which can be resonant with the output field (homodyne detection) or detuned from it (heterodyne detection) $[1,3]$.

In this paper we scrutinize diffusive unravelings of MEs in the context of experimentally demonstrating the detector dependency of quantum dynamics [4]. Historically, before the emergence of quantum trajectory theory $[2,5-$ $8]$, it was widely believed that a system's quantum dynamics was objective, and thus independent of any measurement. This notion was proposed in early works of Einstein on emission and absorption of light quanta by an atom [9] such that the system (atom) state undergoes evolution among stationary states of Bohr [10]. But since the early 90's, modern theory of quantum dynamics has rendered this idea obsolete, implying that dynamical quantum events are subject to a remote detector.

It remains a challenge, however, to verify experimentally the testimony of this theory. In 2012, a proposal was made for an experiment to disprove all objective pure-state dynamical models (OPDM) for a particular quantum system [4]. If an experimentalist had perfect detection (and collection) efficiency, $\eta=1$, the conditioned pure state would be pure, and it would simply be a matter of verifying that two different detection schemes lead to different types of pure states. That is, the problem would be a continuous-in-time version of the Einstein-Podolsky-Rosen (EPR) phenomenon [11]. However, in the real experimental setups $\eta<1$, and in order to rule out all OPDMs one would require to invoke the more general form of the EPR argument known as steering [12] (the term introduced by Schrödinger for this phenomenon [13]), or EPR-steering [14]. In this formalism one experimenter (Alice) proves that the objective quantum state of the other experimenter (Bob) cannot 
be explained by any local hidden state (LHS) models. This is fulfilled by expressing the correlation of measurements of the two parties in terms of an inequality, the violation of which can be interpreted as ruling out all LHS models for Bob's system. Generalizing this to continuous monitoring [4], inequalities can be derived of which the violation would rule out all OPDMs, and consequently confirm the detector-dependency of stochastic quantum dynamical evolutions.

The physical system studied in Ref. [4] was a resonantly driven two-level atom with two measurement arrangements to capture the fluorescence. It turns out that using two homodyne detection, and assuming both have equal efficiencies, the critical efficiency to disprove OPDMs is $\eta_{c} \approx 73 \%$, which is quite high. Further, it was proved [4] that $\eta>50 \%$ is a prerequisite for being able to demonstrate EPR-steerability using any dyne detection schemes. Very recently, two of us have generalized this necessary condition [15], showing that for any Markovian open quantum system with an arbitrary number $L$ of decoherence channels to the environment and with an arbitrary number $K$ of diffusive unravelings, unless at least one member of the set $\left\{\eta_{\ell}^{k}\right\}$ is greater than $50 \%$, the diffusive unravelings cannot be used to demonstrate detector dependency of the conditional state matrix $\varrho$.

The present work furthers the investigation of how well the detector dependency of dynamical quantum events can be proven experimentally using diffusive unravelings, in terms of the efficiency required. Our first aim is to derive even more general necessary conditions, and our second aim is to derive sufficient conditions in a variety of scenarios with a simple system (a qubit).

We organize the paper as follows. First, Sec. II reviews briefly formalism of general diffusive unravelings. In Sec. III it is then shown that the most general proof of a no-go for inefficient diffusion can be cast as a special case of a semidefinite programming (SDP) known as a feasibility problem (FP). We present a simple graphical representation of this general necessary condition for the case where the system interacts with just one environment. Following this, In Sec. IV different MEs and measurement strategies are considered. First we analyze a system with three irreversible channels, and three different diffusive unravelings to try to prove detectordependence. Next, systems with fewer decoherence channels and just two different diffusive unravelings are studied. Finally, in Sec. V we conclude by summarizing the results of this study along with the outcomes of previous publications in Table I.

\section{GENERAL DIFFUSIVE UNRAVELING WITH IMPERFECT DETECTION}

The state $\rho$ of an open quantum system in the Markov approximation, defined by tracing over the environment, has its evolution governed by a ME

$$
\dot{\rho}=-i[\hat{H}, \rho]+\mathcal{D}[\hat{\mathbf{c}}] \rho \equiv \mathcal{L} \rho .
$$

Here $\hat{H}$ is the system Hamiltonian (in units where $\hbar=1$ ), $\hat{\mathbf{c}}=\left(\hat{c}_{1}, \ldots, \hat{c}_{L}\right)^{\top}$ is a vector of arbitrary operators (also know as Lindblad operators [16]), and $\mathcal{D}[\hat{\mathbf{c}}]=\sum_{l=1}^{L} \mathcal{D}\left[\hat{c}_{l}\right]$ where the decoherence superoperator $\mathcal{D}[\hat{o}] \rho=\hat{o} \rho \hat{o}^{\dagger}-$ $1 / 2\left\{\hat{o}^{\dagger} \hat{o}, \rho\right\}$, with curly brackets representing the anticommutator.

The most general diffusive unraveling of the ME, Eq. (1), by allowing for inefficient detection reads [1]

$$
d \varrho=\mathcal{L} \varrho d t+\mathcal{H}\left[d \mathbf{V}^{\dagger} \hat{\mathbf{c}}\right] \varrho,
$$

where $\mathcal{H}[\hat{o}] \rho=\hat{o} \rho+\rho \hat{o}^{\dagger}-\operatorname{Tr}\left[\hat{o} \rho+\rho \hat{o}^{\dagger}\right] \rho$ is a nonlinear superoperator, and $d \mathbf{V}=\left(d V_{1}, \ldots, d V_{L}\right)^{\top}$ is a vector of infinitesimal c-number Wiener increments. These are Gaussian random variables satisfying $\mathrm{E}[d \mathbf{V}]=0$, where $\mathrm{E}[\diamond]$ denotes the ensemble average of the random variable $\diamond$ with respect to its probability distribution function. Physically, they should actually be regarded as noise in the output complex photocurrent

$$
\mathbf{J} d t=\left\langle\Theta \hat{\mathbf{c}}+\Upsilon \hat{\mathbf{c}}^{\ddagger}\right\rangle d t+d \mathbf{V},
$$

with the following correlation relations

$$
d \mathbf{V} d \mathbf{V}^{\dagger}=\Theta d t, \quad d \mathbf{V} d \mathbf{V}^{\top}=\Upsilon d t,
$$

where $\mathbf{o}^{\ddagger}=\left(\mathbf{o}^{\top}\right)^{\dagger}$, and $\langle\hat{o}\rangle=\operatorname{Tr}[\hat{o} \varrho]$ is the quantum mechanical expectation value of an operator $\hat{o}$ with respect to the conditioned quantum state $\varrho$. Here $\Theta=$ $\operatorname{diag}\left(\eta_{1}, \ldots, \eta_{L}\right)$ is a real diagonal matrix allowing for imperfect detection in the formalism so that channel $l$ is monitored with efficiency $0 \leq \eta_{l} \leq 1$. The rest of parameters that characterize diffusive unravelings are being encoded in a complex symmetric matrix $\Upsilon=\Upsilon^{\top}$. It is convenient to encapsulate all of the correlation properties in the so-called unraveling matrix [17]

$$
U(\Theta, \Upsilon) \equiv \frac{1}{2}\left(\begin{array}{cc}
\Theta+\operatorname{Re}[\Upsilon] & \operatorname{Im}[\Upsilon] \\
\operatorname{Im}[\Upsilon] & \Theta-\operatorname{Re}[\Upsilon]
\end{array}\right),
$$

with the only constraint that it must be positive semidefinite (PSD), i.e. that $\exists Z \in \mathbb{C}^{2 L \times 2 L}$ such that $U(\Theta, \Upsilon)=Z^{\top} Z$. Of course, one could work with other matrix representations of diffusive measurements other than $U$ [18], but for the sake of consistency with Ref. [15] we rather keep this notation, even though we shall also give a technical reason why it is not an appropriate choice (see Sec. IIIB). Thus an ideal monitoring of the environment giving rise to a pure-state quantum diffusion is represented by $U(I, \Upsilon)$, where $I \in \mathbb{R}^{L}$ is an identity matrix, and correspondingly Eq. (2) can be replaced with a stochastic Schrdinger equation.

An interesting class of unravelings is obtained when the Lindblad operators are Hermitian $\hat{\mathbf{c}}=\hat{\mathbf{c}}^{\ddagger}$ and the complex Wiener increments satisfy

$$
d V_{l}=e^{i \phi} \sqrt{\eta} d W_{l}, \quad l=1, \cdots, L,
$$

where

$$
d W_{j} d W_{k}=\delta_{j k} d t
$$


Therefore, depending on the monitoring scheme the $l$ th measurement outcome would be either a current containing maximal information about the observable $\hat{c}_{l}$,

$$
J_{l}^{\text {current }} d t=2 \eta\left\langle\hat{c}_{l}\right\rangle d t+\sqrt{\eta} d W_{l} \quad(\phi=0),
$$

or just a pure-noise,

$$
J_{l}^{\text {noise }} d t=i \sqrt{\eta} d W_{l} \quad(\phi=\pi / 2) .
$$

In the first case, the associated conditional evolution tends to localize the system to a $\hat{c}_{l}$ eigenstate:

$$
d \varrho=\mathcal{L} \varrho d t+\sqrt{\eta} \sum_{l=1}^{L}\left[\left(\hat{c}_{l}-\left\langle\hat{c}_{l}\right\rangle\right) \varrho+\text { H.c. }\right] d W_{l} .
$$

In the latter case, the stochastic evolution corresponds to a noisy Hamiltonian, since no information about the system is being obtained:

$$
d \varrho=\mathcal{L} \varrho d t+i \sqrt{\eta} \sum_{l=1}^{L}\left[\varrho, \hat{c}_{l}\right] d W_{l} .
$$

We employ these kind of unravelings in Sec. IV for some specific scenarios.

Now having this formalism in our arsenal we can investigate proving detector dependency of diffusive stochastic evolution.

\section{NECESSARY CONDITIONS FOR DEMONSTRATING DETECTOR DEPENDENCE}

\section{A. Coarse graining}

In addition to the mathematical tools that have been described thus far we require another essential concept, that is, coarse graining of diffusive unravelings. To this end, consider two unravelings $U \equiv U(\Theta, \Upsilon)$ and $U_{0} \equiv$ $U\left(\Theta_{0}, \Upsilon_{0}\right)$ with associated vectors of Wiener processes $d \mathbf{V}$ and $d \mathbf{V}_{0}$, respectively. If it is possible to express the latter as the algebraic sum of the former and another complex vector Wiener increment $d \check{\mathbf{V}}$ obeying the above restrictions, i.e. $d \mathbf{V}_{0}=d \check{\mathbf{V}}+d \mathbf{V}$, then the first unraveling $U$ can be realized experimentally by implementing the second one, $U_{0}$, and keeping just the relevant information in record, discarding the information in $d \mathbf{V}$. This will be so if the tacit unraveling matrix for $d \check{\mathbf{V}}, \check{U} \equiv U_{0}-U$, is also PSD. Under these conditions we call $U$ a coarse graining of $U_{0}$, and $U_{0}$ a fine graining of $U$ (and of $\tilde{U}$ ).

\section{B. Arbitrary number $L$ of environments}

Consider an experimenter who is able to implement a set $\mathfrak{U}$ of some number $K>1$ of distinct unravelings

$$
\mathfrak{U}=\left\{U_{k} \equiv U\left(\Theta_{k}, \Upsilon_{k}\right): U_{k} \geq 0, k=(1, \ldots, K)\right\} .
$$

As pointed out in the introductory section, it is necessary to perform EPR-steering in order to prove that conditional stochastic evolution of the system is determined by a distant detection apparatus. This means that if there exists a single unraveling $U_{0}$ so that $\forall k, \check{U}_{k} \equiv U_{0}-U_{k} \in$ $\mathbb{R}^{2 L \times 2 L}$ is PSD, then every member of the set $\mathfrak{U}$ is a coarse graining of $U_{0}$. If such a $U_{0}$ exists, then $\Theta_{0}$ can always be chosen to equal $I$ (as this just makes every $\tilde{U}_{k}$ more positive), so that $U_{0}$ is a purity-preserving unraveling. In other words, there exists a pure-state dynamical model which could underly all of the different stochastic evolutions induced by the $K$ different measurement schemes, namely that corresponding to $U_{0}$. Consequently, that set $\mathfrak{U}$ cannot possibly be used to rule out all OPDMs.

Thus we have a necessary condition for demonstrating detector dependence: the non-existence of a common fine-graining $U_{0}$ for every member of $\mathfrak{U}$. But is there a way to establish the existence, or non-existence, of such a $U_{0}$ given $\mathfrak{U}$ ?

In Ref. [15] a partial answer was given, in the form of a no-go theorem for inefficient diffusive unravelings: if for all decoherence channels $l$ and for all monitoring schemes $k$ the detection efficiencies satisfy $\eta_{l}^{k} \leq 0.5$, then there exists an unraveling $U_{0}$ from which the set $\mathfrak{U}$ can be obtained by coarse graining. This was proven by considering the choice $U_{0}=U(I, 0)$. This unraveling corresponds to "quantum state diffusion" (QSD) as introduced in Refs. $[19,20]$ (as an objective pure state dynamical model) and could be realized by unit-efficiency heterodyne detection [8].

In this paper we prove a much more general no-go theorem. Rather than considering a particular $U_{0}$, and seeing what that implies, we consider any given set $\mathfrak{U}$ and define a procedure to determine whether any $U_{0}$ exists. The procedure is, in general, numerical rather than analytical, but it can be performed efficiently because of the power of semi-definite programming [22]. To reiterate the problem definition, given a set of diffusive unravelings $\mathfrak{U}=\left\{U\left(\Theta_{k}, \Upsilon_{k}\right)\right\}_{k=1}^{K}$, we want to determine whether there exists a $\Upsilon_{0}$ such that $U\left(I, \Upsilon_{0}\right) \geq 0$ and $\forall k, U\left(I-\Theta_{k}, \Upsilon_{0}-\Upsilon_{k}\right) \geq 0$

\section{Solution via Semi-Definite Programming}

With this formulation in hand, the problem can be formalised as a particular class of semidefinite programming (SDP) which is referred to as a feasibility problem [21], the description of which comes below. The standard definition of a (dual) semidefinite program is [22]

$$
\begin{aligned}
\operatorname{minimize} & \mathbf{b}^{\top} \mathbf{x} \\
\text { subject to } & F(\mathbf{x}) \geq 0,
\end{aligned}
$$

where

$$
F(\mathbf{x})=F^{0}+\sum_{j=1}^{n} \mathbf{x}_{j} F^{j}
$$


Here $\mathbf{b} \in \mathbb{R}^{n}$, and $F_{0}, \cdots, F_{n} \in \mathbb{R}^{m \times m}$ are the known data, and the minimization is over $\mathbf{x} \in \mathbb{R}^{n}$. In terms of these variables, the constraint (14) is a linear matrix inequality (LMI). In the special case in which $\mathbf{b}=0$, the optimization problem reduces to the search for some vector $\mathbf{x}$ satisfying the LMI, which should obviously be an easier computational job in practice. This is an instance of a feasibility problem and is exactly what we need.

To apply the above formalism, we need a one-to-one linear mapping between the symmetric complex $L \times L$ matrix $\Upsilon_{0}$ and a real vector $\mathbf{x} \in \mathbb{R}^{L(L+1)}[23]$

$$
\Upsilon_{0}=\varnothing(\mathbf{x})
$$

Defining $\mathbf{\$}(\mathbf{x})=U(I, \oslash(\mathbf{x}))$, which is still a linear function of $\mathbf{x}$, the required constraints can be written as the LMI (14) by choosing $F(\mathbf{x})$ to be the following blockdiagonal matrix $\in \mathbb{S}^{2 L(K+1)}$ :

$$
F(\mathbf{x})=\left(\begin{array}{c|c|c|c}
\mathbf{\phi}(\mathbf{x}) & 0 & \cdots & 0 \\
\hline 0 & \mathbf{\$}(\mathbf{x})-U_{1} & \cdots & 0 \\
\hline \vdots & \vdots & \ddots & 0 \\
\hline 0 & 0 & \cdots & \mathbf{\$}(\mathbf{x})-U_{k}
\end{array}\right) .
$$

This is because positive semidefiniteness of Eq. (17) ensures that all submatrices must be PSD. Also the linearity of $\mathbf{p}(\mathbf{x})$ and consequently that of $F(\mathbf{x})$ implies that the latter can be written in the form of Eq. (15), with $F^{0} \cdots, F^{n} \in \mathbb{S}^{2 L(K+1)}$, where $n=L(L+1)$. Therefore the task is to find the following feasible set

$$
\mathfrak{F}=\left\{\mathbf{x} \in \mathbb{R}^{L(L+1)}: F(\mathbf{x}) \geq 0\right\} .
$$

Each member $\mathbf{x}$ of this set is called a feasible point, and defines a fine graining $\mathbf{p}(\mathbf{x})$ of every member of the sets $\mathfrak{U}$ as coarse grainings of it. This FP has $2 L(K+1)$ constraints with $L(L+1)$ unknown real parameters. This suggests that other representations of diffusive unravelings which deal with a larger number of unknowns, for example the one introduced in Ref. [18], are not computationally efficient for the particular kind of problems we are considering here. It is known that a SDP can be solved in (approximately) polynomial time with respect to $n=2 L(K+1)$, and with errors as small as one wishes [24]. Thus, finding the unknowns would not be costly from computational viewpoints [25], in particular because no optimization is performed. If it turns out that the set $\mathfrak{F}$ is null then all OPDMs will be ruled out.

This is the no go theorem. It is easiest to see how it works by considering the simple case $L=1$; that is, when the system is allowed to couple with just one environment.
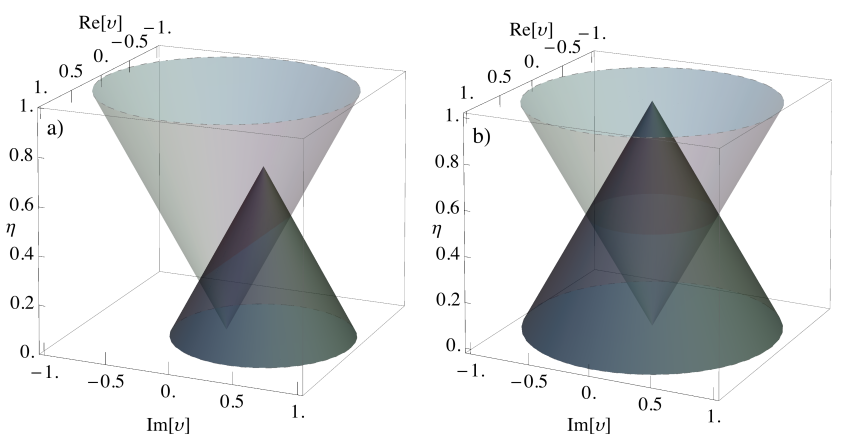

FIG. 1. Feasibility set and space of unravelings for an open quantum system with one environmental decoherence channel $L=1$. The light gray cones represent all physical unravelings that satisfy Eq. (20), and the dark gray cones show unravelings that meet the constraints given in Eq. (21b) for (a) a generic unraveling $U_{0}=U\left(\eta_{0}, v_{0}\right)$, and (b) the QSD unraveling $U_{0}=U(1,0)$. The intersection of these two conical spaces encloses a volume so that all points inside of it can be a coarse graining of the $U_{0}$ located at the vertex.

\section{Single environment $L=1$}

There are numerous physical problems that are modelled using the ME given in Eq. (1) with only one decoherence channel, such as a two-level system with dipolecoupling to a coherent electromagnetic field [26]. In this case the vector of arbitrary operators $\hat{\mathbf{c}}$ in Eq. (2) is replaced with a single Lindblad operator $\hat{c}$ and hence the diffusive unraveling is described by

$$
U(\eta, \Upsilon) \equiv \frac{1}{2}\left(\begin{array}{cc}
\eta+\operatorname{Re}[v] & \operatorname{Im}[v] \\
\operatorname{Im}[v] & \eta-\operatorname{Re}[v]
\end{array}\right),
$$

where $\eta \in \mathbb{R}$ and $v \in \mathbb{C}$ are scalars corresponding to matrices $\Theta$ and $\Upsilon$ in Eq. (5), respectively. It is straightforward to check out the positive semidefiniteness constraint for Eq. (19) by inspecting its spectrum. This constraint translate to

$$
|v| \leq \eta
$$

Also, the same procedure applies to the $K+1$ constraints of the feasibility set, Eq. (18), so that they all can be arranged in the following form

$$
\begin{aligned}
\left|v_{0}\right| & \leq \eta_{0}, \\
\forall k \quad\left|v_{0}-v_{k}\right| & \leq \eta_{0}-\eta_{k} .
\end{aligned}
$$

Since each diffusive unraveling can be uniquely determined by $\left.\left(L^{2}+2 L\right)\right|_{L=1}=3$ variables, it is then possible to visualize a feasible set in three dimensional space such that the coordinates are labeled by $\operatorname{Re}[v], \operatorname{Im}[v]$, and $\eta$. The set of all physically meaningful unravelings that satisfy Eq. (20) is shown in Fig. 1a as the light gray cone. Therefore the set $\mathfrak{U}$ given by Eq. (12) that is supposed to be implemented by an experimenter is represented by 
a set of points $\left\{\left(\eta_{k}, v_{k}\right)\right\}_{k=1}^{K}$ in this cone. Moreover, it is evident that unravelings $U_{0}$ that comply with the condition of Eq. (21a) and eventually make up the feasibility set have to lie somewhere in this conical volume. Now by inspection, for a given $U_{0}$, Eq. (21b) states that the points in $\mathfrak{U}$ lie in an inverted cone whose apex is $\left(\eta_{0}, v_{0}\right)$. Thus, referring to Fig. 1a, the question now is: does there exist a point $\left(\eta_{0}, v_{0}\right)$ in the light gray cone which is the apex of an inverted (dark gray) cone that includes all the points in $\mathfrak{U}$ ?

If we consider $U_{0}=U(1,0)$ (heterodyne detection, or QSD), the two cones intersect each other right at $|v|=\eta=0.5$; see Fig. 1b. It can obviously be seen that a set $\mathfrak{U}$ containing only unravelings with efficiencies $\eta \leq 0.5$ is not capable of demonstrating EPR-steering (as they are confined in the shared volume of the two cones). In other words, to be able to rule out all OPDMs, at least one efficiency has to be greater than 0.5. However, as mentioned in Ref. [15] this is just a necessary condition and is not sufficient to prove the detector dependency of stochastic conditional states $\varrho$. This insufficiency can be seen immediately by looking at the upper half part of Fig. 1b where there are other unravelings in the overlapped volume that are also a coarse graining of $U(1,0)$, those with $|v|<1-\eta<0.5$.

\section{DEMONSTRATING DETECTOR DEPENDENCE}

Having determined necessary conditions for being able to demonstrate detector-dependence using diffusive unravelings, we now turn to sufficient conditions. This will involve considering particular open qubit systems, coupled to one or more environments, with two or more different unravelings, as well as considering various EPRsteering inequalities that might be violated.

$$
\text { A. } L=3, K=3
$$

Consider first an open qubit system with $L=3$ irreversible environmental channels, with the conditional evolution of system's state characterized by the following SME

$$
d \varrho=\sum_{l=1}^{3}\left(\mathcal{D}\left[\sqrt{\gamma} \hat{\sigma}_{l}\right] \varrho d t+\mathcal{H}\left[\sqrt{\gamma} \hat{\sigma}_{l} d V_{l}\right] \varrho\right)
$$

Here $\hat{\sigma}_{l}$ are the usual Pauli operators. In comparison to the notation of Eq. (2), $\hat{H}=0$ in a relavent rotating frame, $\hat{\mathbf{c}}=\sqrt{\gamma}\left(\hat{\sigma}_{1}, \hat{\sigma}_{2}, \hat{\sigma}_{3}\right)$, and $d \mathbf{V}=\left(d V_{1}, d V_{2}, d V_{3}\right)^{\top}$. An unraveling equivalent to the one described by the SME given in Eq. (22) can be realized for a quantum system with one environment (for example, in a circuit QED setup) but by measuring the three Pauli observables concurrently [29, 30].

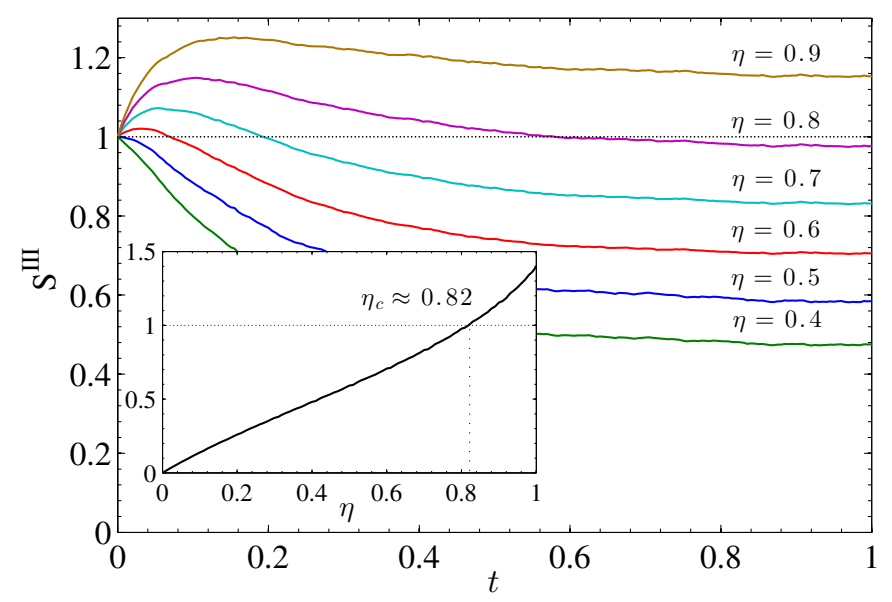

FIG. 2. (Color online). Steering parameter for $K=3$ measurement settings: the temporal behavior of the steering parameter $S^{\mathrm{III}}$, Eq. (23), for different values of the detection efficiency $\eta$. It is evident that all unravelings for which $\eta \leq 0.5$ are not able to demonstrate EPR-steerability. Nevertheless, once the system settles in the steady state $(t \rightarrow \infty)$, it still requires great improvements on detection efficiency to the extent that it should roughly satisfy $\eta>0.8$. Inset shows the value of $S^{\mathrm{III}}$ versus $\eta$ for the system in $\varrho_{\mathrm{ss}}$.

We consider the case where the complex Wiener processes are taken to be in the form of Eq. (6) with $L=3$. Specifically, we consider performing three different unravelings, labelled by $k \in\{1,2,3\}$, in which the experimenter obtains information about $\hat{\sigma}_{k}$ from the by choosing $\phi_{k}=0$ for the $k$ th component of the observable, while giving rise to noisy Hamiltonian evolution for the other components by choosing $\phi_{l}=\pi / 2$ for the $l \neq k$. Every one of these three monitorings can be encapsulated in an unraveling matrix as shown in Eq. (5). For instance, for the $k=3$ unraveling it is simplified to $\Theta=\eta I$, and $\Upsilon=\eta \operatorname{diag}(-1,-1,1)$. The intuition behind this choice is that each unraveling yields information only about one observable, and so would be expected to make this observable take a well-defined value. This would lead to quite different conditioned states for the different unravelings, which is what is needed to disprove all OPDMs.

Thus to prove the detector-dependence of $\varrho$ a suitable EPR-steering criterion should be examined. Such a criterion is often expressed in the form of an inequality. For example, from the fact that the Bloch vector $\mathbf{r}=\left\langle\left(\hat{\sigma}_{1}, \hat{\sigma}_{2}, \hat{\sigma}_{3}\right)^{\top}\right\rangle$ must obey $\|\mathbf{r}\|^{2} \leq 1$, and the convexity of $\left\langle\hat{\sigma}_{l}\right\rangle \equiv \operatorname{Tr}\left[\varrho \hat{\sigma}_{l}\right]$ in the conditional state matrix $\varrho$, it can be shown that every OPDM satisfies $[4,14]$

$$
S^{\mathrm{III}} \equiv \sum_{k=1}^{3} \mathrm{E}^{k}\left[\left\langle\hat{\sigma}_{k}\right\rangle^{2}\right] \leq 1 .
$$

Here $\mathrm{E}^{k}[\bullet]$ means the ensemble average under the unraveling $k$. That is, three unravelings are implemented to calculate the steering parameter $S^{\mathrm{III}}$. Note that due to the symmetrical nature of the problem the ensemble 


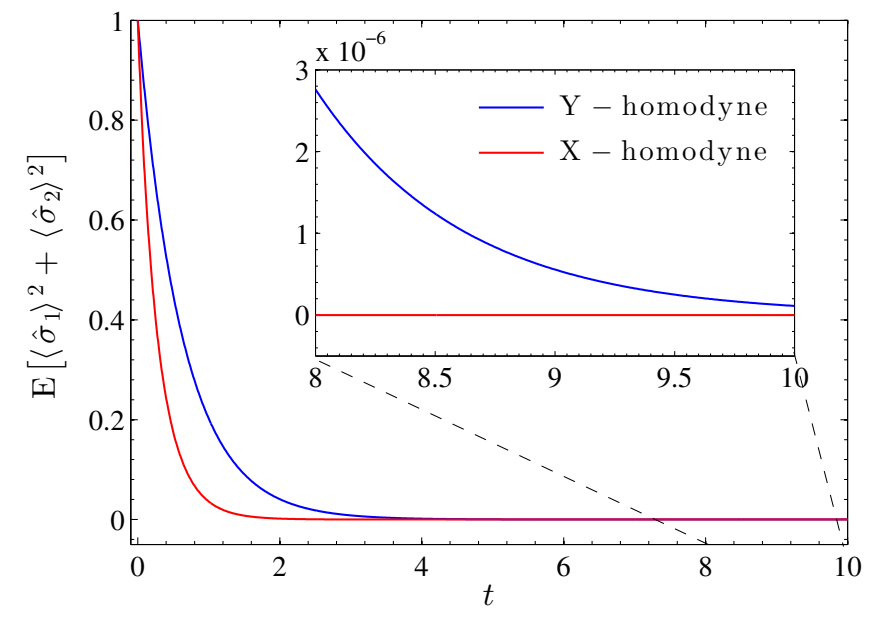

FIG. 3. (Color online). Ensemble average of $\left[\left\langle\hat{\sigma}_{1}\right\rangle^{2}+\left\langle\hat{\sigma}_{2}\right\rangle^{2}\right]$ : results of numerical simulations of the first term in the L.H.S of the inequality given in Eq. (27) using X- and Y-homodyne detection as a function of time for $\eta=0.6$.

average does not depend on $k$ when the system is in the steady state for $t \rightarrow \infty$.

Details of calculations are presented in App. A 1. As a function of time $t$ we plot in Fig. 2 the steering parameter given in Eq. (23) for various values of the detection efficiency $\eta$. It can be clearly seen that as long as $\eta \leq 0.5$ the EPR-steering inequality is never violated. This should not be very surprising as it was comprehensively analyzed in the previous section. Nevetheless, violation occurs while the system is in the transient state provided that $\eta>0.5$. In contrast, once the system relaxes to its steady state (which might be much more interesting from practical points of view) only if $\eta>0.8$ it is possible to establish EPR-steerable states. This is also illustrated in the inset where the value of $S^{\mathrm{III}}$ versus $\eta$ is drawn when the system dynamics reach to a stable point. The critical efficiency of $\eta_{c} \approx 0.82$ is required to prove detector dependency of $\varrho$ in the steady state.

\section{B. $L=1, K=2$}

The high efficiency required by the 3-measurement setting suggests looking at the other extreme in which an open quantum system decoheres just to a single environment. Consider that the SME of such a system is given by

$$
d \varrho=\mathcal{D}\left[\sqrt{\gamma} \hat{\sigma}_{3}\right] \varrho d t+\mathcal{H}\left[\sqrt{\gamma} \hat{\sigma}_{3} d V\right] \varrho,
$$

where again in notation of Eq. (2) Hamiltonian becomes zero in a suitable rotating frame, $\hat{\mathbf{c}}=\sqrt{\gamma} \hat{\sigma}_{3}$, and the Wiener process is given by Eq. (6) with $L=1$. Here we consider just two different measurement strategies, corresponding to $\phi=0$ (X-homodyne, giving maximum information), and $\phi=\pi / 2$ (Y-homodyne, giving pure noise). Since $L=1$, the correlation matrices are just scalars, $\Theta=\eta= \pm \Upsilon$ for the two respective cases.

For the 2-measurement settings we study a class of EPR-steering inequalities similar to the one given in Eq. (23) which in general can be formulated as

$$
\mathrm{E}^{\mathrm{X}}\left[\sum_{k=1}^{3} \alpha_{k}\left\langle\hat{\sigma}_{k}\right\rangle^{2}\right]+\mathrm{E}^{\mathrm{Y}}\left[\sum_{k=1}^{3}\left(1-\alpha_{k}\right)\left\langle\hat{\sigma}_{k}\right\rangle^{2}\right] \leq 1,
$$

where

$$
\forall k, \quad 0 \leq \alpha_{k} \leq 1 .
$$

This shows that there are an infinite number of such inequalities. However, one should carefully choose an optimal one so that the left hand side of Eq. (25) constitutes a maximum. This is determined by whether the $\mathrm{X}$-homodyne or Y-homodyne measurement scheme gives a larger value for the ensemble average of $\left\langle\hat{\sigma}_{k}\right\rangle^{2}$. If the former is the case one should choose $\alpha_{k}=1$, and in the case of the latter $\alpha_{k}=0$. There are protocols in which both $\mathrm{X}$ - and Y-homodyne detection give an equal amount of information about the $k$ th observable. In such a case $\alpha_{k} \in[0,1]$ is arbitrary. Note that the comparison between $\mathrm{E}^{\mathrm{X}}\left[\left\langle\hat{\sigma}_{k}\right\rangle^{2}\right]$ and $\mathrm{E}^{\mathrm{Y}}\left[\left\langle\hat{\sigma}_{k}\right\rangle^{2}\right]$ can be done through either numerical simulation or analytical calculation where possible.

In this work we have chosen the optimal EPR-steering inequalities of the form (25) by analyzing the numerical results. It turns out that for the SME given in Eq. (24) the following inequality is optimal

$$
S^{\mathrm{II}} \equiv \mathrm{E}^{\mathrm{Y}}\left[\left\langle\hat{\sigma}_{1}\right\rangle^{2}+\left\langle\hat{\sigma}_{2}\right\rangle^{2}\right]+\mathrm{E}^{\mathrm{X}}\left[\left\langle\hat{\sigma}_{3}\right\rangle^{2}\right] \leq 1 .
$$

This can be clearly seen in Fig. 3 where the ensemble average of $\left\langle\hat{\sigma}_{1}\right\rangle^{2}+\left\langle\hat{\sigma}_{2}\right\rangle^{2}$ is plotted using both $\mathrm{X}$ - and $\mathrm{Y}$ homodyne monitorings. The same argument holds for the second term of Eq. (27) but this time unraveling using $\mathrm{X}$-homodyne gives more information about $\hat{\sigma}_{3}$ in comparison to Y-homodyne. Each one of these two terms in Eq. (27) can be realized in an an EPR-steering experiment as follows. Bob randomly chooses between the two measurement settings, and so tells Alice to implement either X- or Y- homodyne. Bob then correlates the outcomes of his tomographic measurements with those obtained by Alice. For details, see Ref. [4].

In Fig. 4 the time variation of the steering parameter $S^{\mathrm{II}}$ for different values of detection efficiency $\eta$ is shown. There are two key features closely resembling those of Sec. IV A. Firstly, the necessary condition $\eta>0.5$ for ruling out all OPDMS is satisfied. Secondly, the transient phase of evolution establishes EPR-steering states provided the detection efficiency is greater than 0.5.

However, in a third respect this case is quite different: in the long-time limit it is impossible to prove detector dependency of $\varrho$ no matter how close to unity $\eta$ is. This is because the $\mathrm{Y}$ - homodyne unraveling generates just pure noise, which contains information about the system evolution (the noisy Hamiltonian) but no information about 


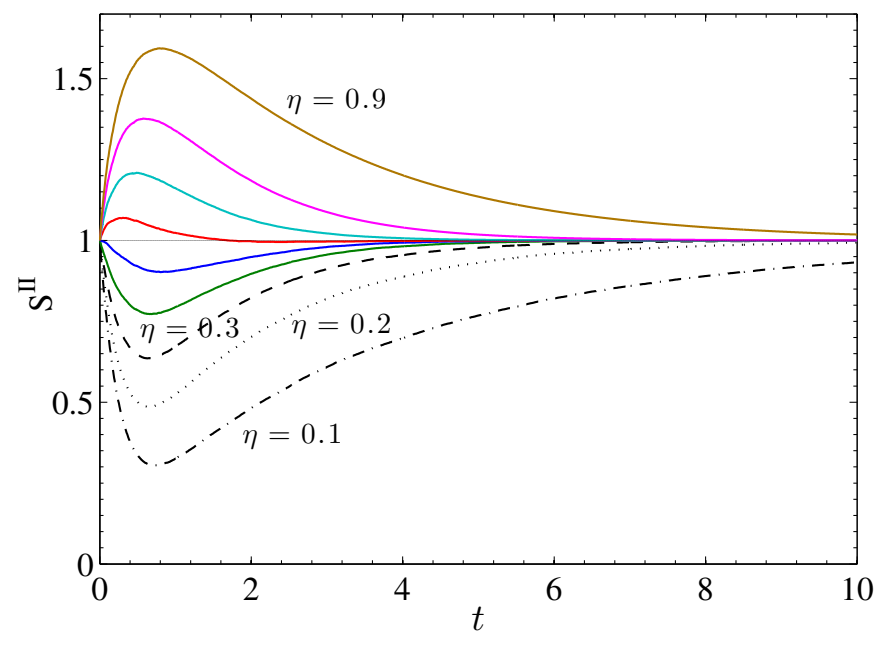

FIG. 4. (Color online). Steering parameter for $K=2$ measurement settings: results of numerical simulations of the steering parameter $S^{\mathrm{II}}$, Eq. (27), as a function of time for different values of $\eta$. Here for the sake of clarity we discard labels in between solid curves, but they follow the same color style and order as in Fig. 2. When the detection efficiencies satisfy $\eta \leq 0.5$ there is no possibilities (for homodyne unravelings) to demonstrate EPR-steering, as expected. However, beyond this limit, it is possible to observe EPR-steering if the system is in its transient phase of evolution. In contradiction to the case of $L=3, K=3$, in the long-time limit there is never any violation of Eq. (27). Curves for $\eta \leq 0.3$ illustrate that the time taken to reach the asymptotic value of 1 grows as the efficiency gets smaller.

the system state. Thus for any $\eta<1$, the system purity decays deterministically over time under the $\phi=\pi / 2$ unraveling, eventually reaching a completely mixed state as $t \rightarrow \infty$. In this limit, the first term in Eq. (27) will be zero, and since the second term is bounded above by unity, there is no way the inequality can be violated. Violations occur at short times only because we started the system in a pure state. Further details are given in App. A 2. It is interesting to note that the transient evolution here spans over an interval several times longer than the duration in which $S^{\text {III }}$ attains steady state.

\section{C. $\quad L=2, K=2$}

Finally, we concisely present the results of our studies of an open qubit system coupled to two irreversible channels $(L=2)$, and compare them to the two results of us previously obtained in Ref. [15]. To this end consider a qubit SME in the form of

$$
d \varrho=\sum_{l= \pm}\left(\mathcal{D}\left[\sqrt{\gamma_{l}} \hat{\sigma}_{l}\right] \varrho+\mathcal{H}\left[\sqrt{\gamma_{l}} \hat{\sigma}_{l} d V_{l}\right] \varrho\right)
$$

where $\hat{\sigma}_{ \pm}=\left(\hat{\sigma}_{1} \pm i \hat{\sigma}_{2}\right) / 2$ are raising and lowering operators. As before, the system's conditional state matrix $\varrho$ evolves in an appropriate rotating frame so that $\hat{H}=0$ in the notation of Eq. (2). Also the Lindblad vector is $\hat{\mathbf{c}}=\left(\sqrt{\gamma_{-}} \hat{\sigma}_{-}, \sqrt{\gamma_{+}} \hat{\sigma}_{+}\right)$, and the independent Wiener processes are given by Eq. (6). Thus, depending on the measurement strategy, determined by the phase term $\phi$ in Wiener process, the average current of the two different decoherence channels would be

$$
\left\langle J_{ \pm}\right\rangle=\eta \sqrt{\gamma_{ \pm}}\left\langle\hat{\sigma}_{ \pm}+e^{i 2 \phi} \hat{\sigma}_{\mp}\right\rangle,
$$

such that the $\mathrm{X}$-homodyne detection $(\phi=0)$ gives information about $\hat{\sigma}_{1}$

$$
\left\langle J_{ \pm}\right\rangle^{X}=\eta \sqrt{\gamma_{ \pm}}\left\langle\hat{\sigma}_{1}\right\rangle
$$

and the outcome of the Y-homodyne detection $(\phi=\pi / 2)$ is given by

$$
\left\langle J_{ \pm}\right\rangle^{Y}= \pm i \eta \sqrt{\gamma_{ \pm}}\left\langle\hat{\sigma}_{2}\right\rangle .
$$

This SME has been studied in Ref. [15] in order to propose an experimental test for disproving all OPDMs using quantum jumps unravelings for experimental setups with detection efficiencies less than $50 \%$. In this protocol, however, Alice should be able to realize $n$ measurement settings in the azimuthal direction $\varphi \in\{(j / n) \pi\}_{j=1}^{n}$ and one in the $z$ direction to be able to work out the considered EPR-steering inequality [15]

$$
\frac{1}{n} \sum_{j=1}^{n} \mathrm{E}^{\varphi_{j}}\left[\left|\left\langle\hat{\sigma}_{\varphi_{j}}\right\rangle\right|\right]-f(n) \mathrm{E}^{z}\left[\sqrt{1-\left\langle\hat{\sigma}_{3}\right\rangle^{2}}\right] \leq 0 .
$$

Here $\hat{\sigma}_{\varphi}=\hat{\sigma}_{-} e^{i \varphi}+\hat{\sigma}_{+} e^{-i \varphi}$, and the function $f(n)$ is given in Ref. [31] which monotonically decreases with $n$, asymptoting to $2 / \pi$ as $n \rightarrow \infty$. Thus in the limit of large $n$ a smaller detection efficiency is required to demonstrate detector dependence of the conditional state of an open quantum system. It turns out that the critical efficiency is approximately $\eta_{c} \approx 0.59$ provided that one is capable of performing an infinite number of monitoring schemes, and in addition the condition $R \equiv \gamma_{+} / \gamma_{-} \ll 1$ is satisfied. These assumptions are nontheless not very convenient. The former is not practical as it requires great number of measurement settings and the latter leads to a very small violation in comparison with the maximum possible violation when the detection is perfect and $R=1$ [15]. However, it was shown that with just a finite number of measurement settings, $K=n+1=5$, one would be able to have a decent (0.05) violation of Eq. (32) with $\eta \approx 0.78$. In what follows we show that an even more suitable EPR-steering inequality can be derived to rule out all OPDMs with a sufficient condition as close as possible (from above) to the necessary condition bound of $50 \%$.

Thus for the case given by Eq. (28), because the $\hat{\sigma}_{ \pm}$are not Hermitian, both the X-and Y-homodyne measurements yield information about the system, but about different observables, namely $\hat{\sigma}_{1}$ and $\hat{\sigma}_{2}$ respectively, given in Eqs. (30) and (31). This suggests yet another variation derivable from the inequality $\|\mathbf{r}\|^{2} \leq 1$, namely the 
following EPR-steering inequality

$$
S_{ \pm}^{\mathrm{II}} \equiv \mathrm{E}^{\mathrm{X}}\left[\left\langle\hat{\sigma}_{1}\right\rangle^{2}+\frac{\left\langle\hat{\sigma}_{3}\right\rangle^{2}}{2}\right]+\mathrm{E}^{\mathrm{Y}}\left[\left\langle\hat{\sigma}_{2}\right\rangle^{2}+\frac{\left\langle\hat{\sigma}_{3}\right\rangle^{2}}{2}\right] \leq 1
$$

which is a special case of Eq. (25) with $\alpha_{1}=1, \alpha_{2}=0$ and $\alpha_{3}=1 / 2$. This assignment should be now obvious considering the argument made in Sec. IV B and recalling Eqs. (30) and (31). In fact the value of $\alpha_{3}$ is arbitrary as both $\mathrm{X}$ - and Y-homodyne give the same amount of information about $\hat{\sigma}_{3}$. The choice $\alpha_{3}=1 / 2$ emphasizes the symmetry.

For sufficiently small $R \ll 1$ it is easy to derive an analytical relation for the steering parameter in Eq. (33),

$$
S_{ \pm}^{\mathrm{II}} \cong 1+8(\eta-0.5) R
$$

The details of the calculation are presented in App. A 3. The analytic relation, Eq. (34), cries out not only the necessary condition mentioned in the Sec. III, but also shows that $\eta>0.5$ is sufficient to rule out all OPDMs. However, this result holds only for small $R$ 's and as such it will not be convincible enough from experimental viewpoint due to a very small violation. Therefore, a desired objective would be to achieve as low as possible to $\eta_{c} \approx 0.5$ and obtain a decent violation of Eq. (33) so that realizing experimentally EPR-steerable states, and hence To this end, for a decent violation of Eq. (33) an optimal value of $R$ is obtained. For example, a few percent of the difference between the threshold and the maximum possible value allowed by quantum mechanics (here unity and 2, respectively) should be almost enough to take into account imperfections. It turns out that an efficiency of approximately 0.72 is needed to acquire a $5 \%$ violation with the ratio of transition rates being optimized at $R \approx 0.2$, see Fig. 5 . In this figure we plot $S_{ \pm}^{\mathrm{II}}$ as a function of $\eta$ with the critical efficiency $\eta_{c}=0.68$ for violating Eq. (33). Also, the inset (bottom-right) depicts the same steering parameter versus $R$ for a fixed detection efficiency $\eta=0.72$.

Finally, one might question the validity of approximations made to obtain Eq. (34), in the sense that it is implying that $\eta>0.5$ should be sufficient for disproving all OPDMs in the limit where $R$ is very small. To support these simplifications which led us achieve the above mentioned analytic formula we present the outcome of numerical simulations of Eq. (33) for $R \approx 0.01$ in the inset (top-left) of Fig. 5. As it can be seen $S_{ \pm}^{\mathrm{II}}$ varies linearly with respect to $\eta$ and this is exactly the behavior one would expect from Eq. (34). Moreover, this plot demonstrates that the critical efficiency is $\eta_{c} \approx 50.7 \%$ which is very close to what analytical calculation predicts, that is $50 \%$. This is a very good improvement on the critical efficiency required for proving detector dependence of stochastic conditional states $\varrho$.

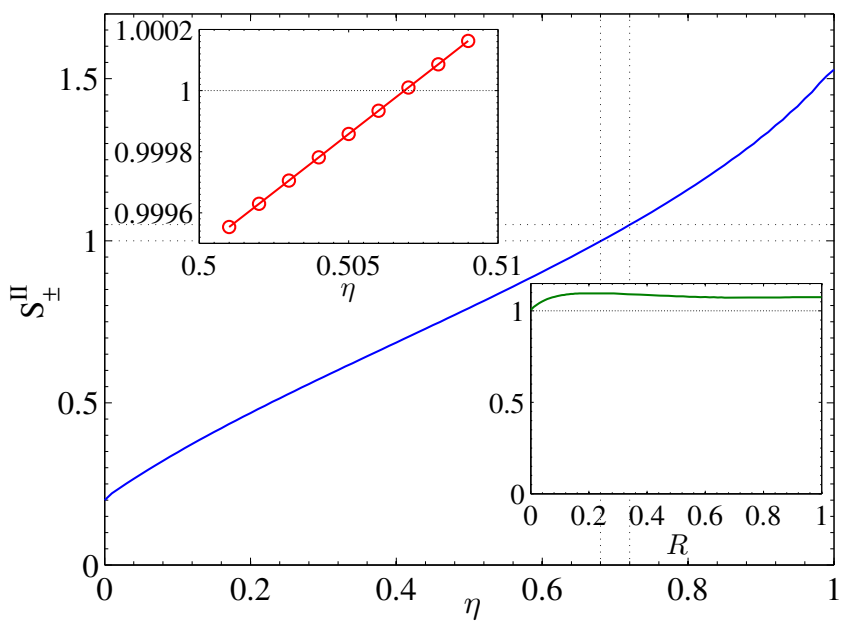

FIG. 5. (Color online). Steering parameter for $K=2$ measurement settings and $L=2$ decoherence channels into the environment: we plot $S_{ \pm}^{\mathrm{II}}$, Eq. (33), as a function of $\eta$ for an optimal value of $R \approx 0.2$ with a significant violation of $5 \%$ at $\eta=0.72$; see text for details. Critical efficiency here is $\eta_{c}=0.68$. Inset: top-left shows the same plot but for $R \approx 0.01$ with critical efficiency of $\eta_{c} \approx 0.507$; bottom-right displays variation of $S_{ \pm}^{\mathrm{II}}$ versus $R$ for $\eta=0.72$.

\section{CONCLUSION}

In summary, we have investigated the dependence of diffusive unravelings of open quantum systems on the existence of a distant detector. Due to imperfect detection schemes some different measurement strategies on an open qubit system were studied to obtain a minimum quantum efficiency required to disprove objectiveness of pure-state evolutionary models. In this work we sought two objectives. Firstly, to determine some general necessary conditions for proving detector dependency of dynamical quantum events. And secondly, to derive sufficient conditions by considering different protocols.

We derive necessary conditions for being able to demonstrate EPR-steering in the form of a no-go for inefficient diffusive unravelings. These conditions depend only on the parameters of the unravelings, not on any details of the system. This theorem can be applied in its most general form by recasting the condition as a special type of semidefinite programming known as a feasibility problem. The simple case of $L=1$ can be thoroughly analyzed by a graphical representation involving right cones.

In contrast to the case of necessary conditions, deriving sufficient conditions can only be done case by case, in that they depend on the systems being examined. To achieve this goal three factors may be inspected for an open qubit system: varieties of irreversible evolution equations, different measurement schemes, and various EPR-steering inequalities. The threshold efficiencies required $\eta_{c}$ for each scenario along with those of other previously proposed experimental tests (for diffusive unravelings) are 
TABLE I. The critical efficiencies $\eta_{c}$ required for violating different EPR-steering criteria. Except for the last two rows, the rest represent entirely different physical systems coupled to different number of decoherence channels; dissipative (Diss) and non-dissipative (ND). Also for each case specific measurement schemes are considered.

\begin{tabular}{cccc}
\hline \hline $\begin{array}{c}\text { Decoherence } \\
\text { channels }(L)\end{array}$ & $\begin{array}{c}\text { Measurement } \\
\text { settings }(K)\end{array}$ & $\begin{array}{c}\text { Steering } \\
\text { parameter }\end{array}$ & $\begin{array}{c}t \rightarrow \infty \\
\eta_{c}\end{array}$ \\
\hline 3 (ND) & 3 & $S^{\mathrm{III}}$, Eq. (23) & 0.82 \\
1 (ND) & 2 & $S^{\mathrm{II}}$, Eq. (27) & - \\
1 (Diss) & 2 & Eq.(3) of Ref. [4] & 0.73 \\
2 (Diss) & 2 & $S_{ \pm}^{\mathrm{II}}$, Eq. (33) & 0.5 \\
2 (Diss) & 2 & Eq.(8) of Ref. [15] & 0.59 \\
\hline \hline
\end{tabular}

summarized in Table I. The investigated environmental decoherence channels fall into two categories; dissipateve (Diss) and non-dissipative (ND). According to the results obtained in this work, the former class shows better figures for the critical efficiency. Among all of these the case in which the system decoheres into two environments and the latter undergo monitoring via two measurement settings (Sec. IV C) shows a promising candidate for being probed empirically. Lastly, this work may, on the one hand, encourage experimentalists to endeavour to increase the efficiencies of detection schemes, and, on the other hand, stimulate theorists to invent still more robust EPR-steering tests.

\section{ACKNOWLEDGEMENT}

This research was supported by the ARC Centre of Excellence Grant No. CE110001027.

\section{Appendix A: Simulating averages}

$$
\text { 1. } L=3, K=3
$$

For the three measurement settings general dyne unraveling as mentioned in the main text, depending on the measurement strategy, Eq. (5) is written in the following forms

$$
\begin{aligned}
& U_{\left.\right|_{k=1}}=\eta \operatorname{diag}(1,0,0,0,1,1), \\
& U_{\left.\right|_{k=2}}=\eta \operatorname{diag}(0,1,0,1,0,1), \\
& U_{\left.\right|_{k=3}}=\eta \operatorname{diag}(0,0,1,1,1,0),
\end{aligned}
$$

which are clearly PSD. Then the conditional state of the system which evolves according to Eq. (22) can be rewritten in the Bloch representation so that dynamics of the system is governed by the following multi-dimensional stochastic differential equation (MDSDE)

$$
d \mathbf{r}=\mathbf{A}(\mathbf{r}) d t+\mathbf{B}(\mathbf{r}) d \mathbf{W}(t),
$$

where

$$
\begin{aligned}
\mathbf{r} & =(x, y, z)^{\top} \equiv\left(\left\langle\hat{\sigma}_{1}\right\rangle,\left\langle\hat{\sigma}_{2}\right\rangle,\left\langle\hat{\sigma}_{3}\right\rangle\right)^{\top}, \\
\mathbf{W} & =\left(W_{1}, W_{2}, W_{3}\right)^{\top}, \\
\mathbf{A} & =-4 \mathbf{r},
\end{aligned}
$$

and the $W_{j}$ s are independent Wiener processes. The matrix $\mathbf{B}$ also takes the following form

$$
\mathbf{B}=2 \sqrt{\eta}\left[\begin{array}{ccc}
0 & -z & -x z \\
z & 0 & -y z \\
-y & x & 1-z^{2}
\end{array}\right]
$$

Here, we have just expressed details of Eq. (A.4) for the $k=3$ case. Note that the ultimate goal is to calculate the steering parameter $S^{\mathrm{III}}$, given in Eq. (23), composed of ensemble averages. By virtue of symmetrical nature of the problem the ensemble average $\mathrm{E}^{k}\left[\left\langle\hat{\sigma}_{k}\right\rangle^{2}\right]$ is independent of $k$ when the system evolves, for a sufficiently long time. Also note that the $k$ th measurement strategy merely gives noisy output for the rest of observables. That is, $\mathrm{E}^{k}\left[\left\langle\hat{\sigma}_{k^{\prime}}\right\rangle^{2}\right]=0$ for $k^{\prime} \neq k$.

As it was discussed in Sec. IV A the $k$ th unravelling of the SME, Eq. (22), gives only information about the $\hat{\sigma}_{k}$ observable. Therefore, to calculate $\mathrm{E}^{k}\left[\left\langle\hat{\sigma}_{k}\right\rangle^{2}\right]$ in Eq. (23) it is convenient to introduce some variables $\beta, \theta$ and $\gamma$ with the following relations

$$
\begin{aligned}
\left\langle\hat{\sigma}_{m}\right\rangle+i\left\langle\hat{\sigma}_{n}\right\rangle & =\sqrt{\beta} e^{i \theta}, \text { for } m, n \neq k \\
\left\langle\hat{\sigma}_{k}\right\rangle^{2} & =\gamma .
\end{aligned}
$$

This allows us to work with a MDSDE which is more relevant to what we need to work out Eq. (23). The following lists different matrices that characterize such a MDSDE for $k=3$

$$
\begin{aligned}
& \underline{\mathbf{r}}=(\beta, \theta, \gamma)^{\top}, \\
& \underline{\mathbf{W}}=\left(W_{\beta}, W_{\theta}, W_{\gamma}\right)^{\top} \text {, }
\end{aligned}
$$

and

$$
\begin{aligned}
& \mathbf{A}=\left[\begin{array}{c}
8 \eta \gamma+4 \eta \beta \gamma-8 \beta \\
\frac{1}{2} \eta \gamma^{2} \sin (4 \theta) \\
4 \eta \beta+4 \eta(1-\gamma)^{2}-8 \gamma
\end{array}\right] \\
& \mathbf{B}=4 \sqrt{\eta}\left[\begin{array}{ccc}
-\sqrt{\beta \gamma} & 0 & -\beta \sqrt{\gamma} \\
0 & \sqrt{\gamma /(4 \beta)} & 0 \\
\sqrt{\beta \gamma} & 0 & \sqrt{\gamma}(1-\gamma)
\end{array}\right]
\end{aligned}
$$

Therefore, to evaluate $S^{\mathrm{III}}$ in Eq. (23) the ensemble average of $\gamma$ for each individual unraveling, $\mathrm{E}^{k}\left[\left\langle\hat{\sigma}_{k}\right\rangle^{2}\right]$, should be calculated. As it follows from Eqs. (A.11)(A.14), the evolution of $\gamma$ in turn is coupled to that of $\beta$ and is independent of variations of $\theta$. Thus, a set of just two coupled SDEs for $\beta$ and $\gamma$ is simulated. This MDSDE is solved numerically using the Milstein method [32]. For sufficiently long time that the system is in the steady state data are sampled and the ensemble averages are calculated. 


$$
\text { 2. } L=1, K=2
$$

The unraveling matrix based upon homodyne measurements on the output field of the system described by Eq. (24) becomes $U=\eta \operatorname{diag}(1,0)$. Then the stochastic equations of motion in the notation of Eqs. (A.4)-(A.6) can be expressed as

$$
\mathbf{A}=-2\left[\begin{array}{l}
1 \\
1 \\
0
\end{array}\right], \quad \mathbf{B}=2 \sqrt{\eta}\left[\begin{array}{ccc}
0 & 0 & -x z \\
0 & 0 & -y z \\
0 & 0 & 1-z^{2}
\end{array}\right]
$$

Now using Eqs. (A.9)-(A.12), this MDSDE is transformed into

$$
\begin{aligned}
& \mathbf{A}=4 \eta\left[\begin{array}{c}
\gamma \beta-\beta / \eta \\
\frac{\gamma}{8} \sin (4 \theta) \\
(1-\gamma)^{2}
\end{array}\right], \\
& \mathbf{B}=4 \sqrt{\eta \gamma}\left[\begin{array}{ccc}
0 & 0 & -\beta \\
0 & 1 / \sqrt{4 \beta} & 0 \\
0 & 0 & 1-\gamma
\end{array}\right] .
\end{aligned}
$$

The same argument as the three measurement settings holds here where the system dynamics at any given time can be described by variables $\beta(t)$ and $\gamma(t)$, and also the same procedure is employed to calculate the ensemble average of $\left[\left\langle\hat{\sigma}_{3}\right\rangle^{2}\right]$.

For the noisy Hamiltonian evolution measurement scheme though, the unraveling matrix is $U=\eta \operatorname{diag}(0,1)$. The ensemble average of $\left[\left\langle\hat{\sigma}_{1}\right\rangle^{2}+\left\langle\hat{\sigma}_{2}\right\rangle^{2}\right]$, which is equal to that of $[\beta]$ through Eq. (A.9), is obtained by solving the following SDEs

$$
\begin{aligned}
& d \beta=4 \beta(\eta-1) d t \\
& d \gamma=0
\end{aligned}
$$

which lead to the equations of motion

$$
\begin{aligned}
& \beta(t)=\beta(0) e^{4(\eta-1) t} \\
& \gamma(t)=\gamma(0) .
\end{aligned}
$$

$$
\text { 3. } L=2, K=2
$$

In this scenario the homodyne detections (with phases $\phi$ and $-\phi$ ) of the two environmental channels gives the following correlation matrices

$$
\Theta=\operatorname{diag}(\eta, \eta), \quad \Upsilon=\eta \operatorname{diag}\left(e^{-2 i \phi}, e^{2 i \phi}\right) .
$$

These diffusive unravelings yield information only about observables $\hat{\sigma}_{1}(\phi=0)$ and $\hat{\sigma}_{2}(\phi=\pi / 2)$. In fact, one of the reasons why we are working with the specific EPRsteering inequality given in Eq. (33) is because there is not an appropriate unraveling to be useful for calculating the ensemble average of $\left\langle\hat{\sigma}_{3}\right\rangle^{2}$. However, as it is the case here, one could still estimate it from the measurement record for $\hat{\sigma}_{1}$ and $\hat{\sigma}_{2}$.

Due to the symmetry of the problem both terms in the L.H.S of Eq. (33) return the same value. This means we can evaluate one and double it. Thus, the case of Xhomodyne with $\phi=0$ is studied. The dynamics of the qubit is confined to x-z plane such that at any instant of time its state can be described by a point $(x(t), 0, z(t))$ in the Bloch sphere, where the Bloch vector is $\mathbf{r}=(x, y, z)$. The trajectory of this point in the sphere is tracked by solving the coupled SDEs

$$
\begin{aligned}
d x= & -\left(\gamma_{\Sigma} / 2\right) x d t+\sqrt{\eta \gamma_{-}}\left(1+z-x^{2}\right) d W_{-}+ \\
& \sqrt{\eta \gamma_{+}}\left(1-z-x^{2}\right) d W_{+}, \\
d z= & \left(-\gamma_{\Sigma} z+\gamma_{\Delta}\right) d t-\sqrt{\eta \gamma_{-}} x(1+z) d W_{-}+ \\
& \sqrt{\eta \gamma_{+}} x(1-z) d W_{+},
\end{aligned}
$$

where $\gamma_{\Sigma}=\gamma_{+}+\gamma_{-}$and $\gamma_{\Delta}=\gamma_{+}-\gamma_{-}$. As we have done for previous simulating jobs we use Milstein method to numerically compute these equations. Once the system reaches to its steady state, $t \rightarrow \infty$, data is being recored to calculate $\mathrm{E}^{\mathrm{X}}\left[\left\langle\hat{\sigma}_{2}\right\rangle^{2}+1 / 2\left\langle\hat{\sigma}_{3}\right\rangle^{2}\right]$, and hence $\mathrm{E}^{\mathrm{Y}}\left[\left\langle\hat{\sigma}_{1}\right\rangle^{2}+1 / 2\left\langle\hat{\sigma}_{3}\right\rangle^{2}\right]$, for each measurement scheme as described above.

Finally, when $R \equiv \gamma_{+} / \gamma_{-} \ll 1$, the conditional state of the system under diffusive unraveling is almost in proximity of the ground state so that approximations $x=O(\sqrt{R})$ and $1+z=O(R)$ hold. Then Eqs. (A.23) and (A.24) are transformed, to leading order, to

$$
\begin{aligned}
& d x=-(\gamma / 2) x d t+2 \sqrt{\eta \gamma R} d W_{+}, \\
& d z=\gamma[2 R-z-1] d t+2 \sqrt{\eta \gamma R} x d W_{+},
\end{aligned}
$$

where $\gamma=\gamma_{-}$. It is straightforward to calculate the ensemble averages $\mathrm{E}\left[x^{2}\right] \approx 4 \eta R$ and $\mathrm{E}\left[z^{2}\right] \approx 1-4 R$ for the steady state. Plugging these results back into Eq. (33) it is easy to show that for small $R$ it is approximated by

$$
S_{R \ll 1} \cong 1+8(\eta-0.5) R .
$$

[1] H. M. Wiseman and G. J. Milburn, Quantum Measurement and Control Cambridge Univ. Press, Cambridge, (2010).
[2] H. J. Carmichael, An Open Systems Approach to Quantum Optics Springer, Berlin (1993).

[3] H. A. Bachor and T. C. Ralph, A Guide to Experiments 
in Quantum Optics Wiley-VCH Verlag, Weinheim (2004)

[4] H. M Wiseman and J. M. Gambetta Phys. Rev. Lett. 108, 220402 (2012)

[5] J. Dalibard, Y. Castin, and K. Mølmer Phys. Rev. Lett. 68, 580 (1992).

[6] C. W. Gardiner, A. S. Parkins, and P. Zoller Phys. Rev. A 464363 (1992).

[7] A. Barchielli Int. J. Theor. Phys. 322221 (1993).

[8] H. M. Wiseman and G. J. Milburn, Phys. Rev. A 47, 1652 (1993).

[9] A. Einstein Phys. Z. 18, 121 (1917).

[10] N. Bohr Philos. Mag. 26, 1 (1913).

[11] A. Einstein, B. Podolsky, and N. Rosen, Phys. Rev. 47, 777 (1935).

[12] H. M. Wiseman, S. J. Jones, and A. C. Doherty, Phys. Rev. Lett. 98, 140402 (2007).

[13] E. Schrödinger, Proc. Cambridge Philos. Soc. 31, 553 (1935).

[14] E. G. Cavalcanti, S. J. Jones, H. M. Wiseman, and M. D. Reid Phys. Rev. A 80032112 (2009)

[15] S. Daryanoosh and H. M. Wiseman New J. Phys. 16, 063028 (2014)

[16] G. Lindblad, Commun. Math. Phys. 48, 119 (1976).

[17] H. M. Wiseman and L. Diósi, Chem. Phys. 268, 91 (2001).

[18] A. Chia and H. M. Wiseman, Phys. Rev. A 84, 012119 (2011).

[19] N. Gisin and I. C. Percival J. Phys. A 25, 5677 (1992)

[20] N. Gisin and I. C. Percival Phys. Lett. A 167, 315-318 (1992)

[21] P. A. Parrilo and S. Lall, Euro. J. Control 9, 307-321 (2003).
[22] L. Vandenberghe and S. Boyd, SIAM Rev. 38(1), 49-95 (1996).

[23] Note that one could encode both matrices $\Upsilon_{0}$ and $\Theta_{0}$ in the vector $\mathbf{x}_{0}$. However, this not only expands the size of unknowns by $L$, which is undesirable, but also gives no additional information in comparison with the hypothetical case of $\Theta_{0}=I$. That is, if there does not exit an unraveling $U\left(I, \Upsilon_{0}\right)$ by which all other unravelings can be coarse grained, then it would be entirely pointless to add another layer of complexity to the problem by considering $\Theta_{0} \neq I$.

[24] B. Gärtner and J. Matoušek, Approximation Algorithms and Semidefinite Programming Springer-Verlag, Berlin, Heidelberg (2012).

[25] B. Kulis, S. Sra and I. Dhillon, JMLR W\&CP 5, 296-303 (2009).

[26] H. Mabuchi and A. C. Doherty Science 298, 5597, 13721377 (2002).

[27] K. W. Murch, S. J. Weber, C. Macklin, and I. Siddiqi, Nature. 502, 211 (2013)

[28] G. de Lange, D. Riste, M. J. Tiggelman, C. Eichler, L. Tornberg, G. Johansson, A. Wallraff, R. N. Schouten, and L. DiCarlo Phys. Rev. Lett. 112, 080501 (2014)

[29] R. Ruskov, A. N. Korotkov, and K. Mølmer, Phys. Rev. Lett 105100506 (2010)

[30] R. Ruskov, J. Combes, K. Mølmer, and H. M. Wiseman Phil. Trans. R. Soc. A 3705291 (2012)

[31] S. J. Jones and H. M. Wiseman, Phys. Rev. A 84, 012110 (2011).

[32] P. E. Kloeden and E. Platen Numerical Solution of Stochastic Differential Equations, Berlin Heidelberg: Springer-Verlag (1992) 